\title{
Using a Thematic Model to Enrich Photo Montages
}

\author{
Charlie Hargood \\ LSL \\ ECS, Southampton University \\ Southampton, United Kingdom \\ cah07r@ecs.soton.ac.uk
}

\author{
David E Millard \\ LSL \\ ECS, Southampton University \\ Southampton, United Kingdom \\ dem@ecs.soton.ac.uk
}

\author{
Mark J Weal \\ IAM/LSL \\ ECS, Southampton University \\ Southampton, United Kingdom \\ mjw@ecs.soton.ac.uk
}

\begin{abstract}
Narrative systems attempt to present users with media collections that include some element of structure or story, however these collections can lack an authorial voice and seem bland as a result. In this paper we explore how themes could be used to enrich automatically generated narratives, and describe how a system which generated story selections in the form of photo montages was developed using a thematic model of narrative. This was achieved by selecting narrative atoms, in this case photographs, from a selection of images on a specific subject with relevance to a desired theme. Our pilot study shows that our thematic system selects images with greater relevance to desired titles, and that the positive impact of thematic selection increases when the images are presented together. We hope that our thematic work will inform others working on narrative systems, and will lead to richer automated narratives.
\end{abstract}

\section{Categories and Subject Descriptors}

H.1 [Models and Principles]: General

\section{General Terms}

Standardization, Human Factors, Experimentation

\section{Keywords}

Narrative, Narrative Generation, Thematics, Folksonomies

\section{INTRODUCTION}

We live in a world of rich media collections, whether it be professionally produced material from news sources and media outlets, or user generated content, such as shared photos, personal websites and blogs. These collections are large and numerous enough to create a problem of information overload [5].

It is possible to use metadata (such as tags) and other index information (such as link analysis) to filter the information that is given to people. However, while filtered

Permission to make digital or hard copies of all or part of this work for personal or classroom use is granted without fee provided that copies are not made or distributed for profit or commercial advantage and that copies bear this notice and the full citation on the first page. To copy otherwise, to republish, to post on servers or to redistribute to lists, requires prior specific permission and/or a fee.

HT'09, June 29-July 1, 2009, Torino, Italy.

Copyright 2009 ACM 978-1-60558-486-7/09/06 ...\$5.00. results can produce individual items of appropriate quality, the overall set of items given to the user can lack cohesion; especially when viewed in contrast to our normal way of communicating complex subjects, through narrative constructs such as news stories, journal accounts, picture books and articles.

Narrative is potentially a very powerful way of presenting human experience as stories that can engage and entertain those consuming the information. The field of narrative generation seeks to develop ways for machines to automatically create narratives, and to improve on current filtering techniques. However, existing narrative generation techniques can produce bland and unengaging narratives that, while communicating the information and experience that makes up the story, lack the engaging and rich experience that is afforded by human constructed stories.

We propose that narrative generation could be improved by the inclusion of a thematic system that enriched the generated narratives with defined themes and motifs [8]. Our idea is that consistent themes running through a collection will give the materials in it a purpose beyond their place in that collection, so that the whole will be greater than its parts.

In this paper we present an evaluation of a prototype thematic system, based on the thematic model that we have developed. In the evaluation Flickr ${ }^{1}$ is used as a suitable source of narrative elements from which to generate montages, flickr is a good source as it includes manual meta-data (tags) that give us information about the content of images. The purpose of our evaluation is to quantify the thematic model's impact on the quality of the montages by comparing it to keyword selection. Our hope is that our thematic work will lead to better narrative generation techniques in the future.

\section{BACKGROUND}

\subsection{Narratology}

Although narratology, as a study of literature, is mostly focused on the analysis of narrative it provides a detailed insight into how narratives are built.

One approach to narratology, structuralism, deconstructs narrative and aims to learn about the components from which a story is built and how they are connected and contrasted against each other. As this defines tangible objects within a narrative that can be modeled there is much narrative generation can use from structuralism as it can seek

\footnotetext{
${ }^{1}$ http://www.flickr.com
} 
to generate the structures that structuralists have defined. Most structuralist theories asserts that a narrative is composed of any series of human experiences [10], and may be deconstructed into a story and a discourse [4] where the story (or fabula) represents a chronology of all the information to be communicated and the discourse (or sjuzhet) represents what parts of the story are told and how those parts are presented (shown in Figure 1).

The story element is constructed by the experiences that make up the subject of the narrative. In a virtual collection of resources the story represents the collection of experiences represented as resources. The discourse however represents what parts of the story are told (the story selection) and how it is told (the story presentation); if the collection is the story then the result of narrative generation (telling the story) is the discourse.

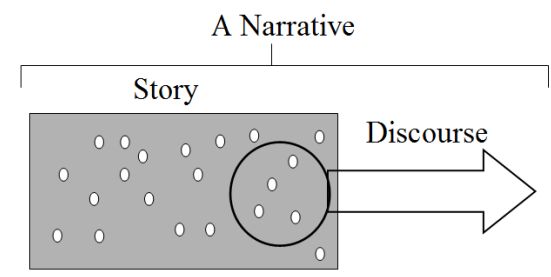

Figure 1: A narrative can be deconstructed into story and discourse

The discourse is the result of a multitude of different mechanics including how the story is presented, what medium is used, the style, the genre, and the themes of the narrative. The study of thematics approaches themes with a structuralist method of deconstruction and attempts to identify the narrative elements that communicate themes.

Tomashevsky deconstructs thematic elements into themes (broad ideas such as 'politics' or 'drama') and motifs (more atomic elements directly related to the narrative such as 'the helpful beast' or 'the thespian') [12]. He describes a structure of themes being built out of sub-themes and motifs. A motif is the smallest atomic thematic element and refers to an individual element within the narrative which connotes in some way the theme. Themes may always be deconstructed into other themes or motifs whereas a motif may not be deconstructed.

\subsection{Narrative Generation}

Narrative generation has a variety of applications in systems that deal with different information, as a narrative can be any collection of human experience it is not limited to written prose but to any representation of human experience. Some systems use narrative as a lens through which to view a larger collection, for example PhotoCopia [13] which presents narrative photo montages. Some systems generate narratives to add more meaning to information, for example Topia [3] where search results are presented as a discourse. Using narrative as a representation of information in this way is similar to various hypertext projects such as AHA! [7] where the omission, emphasis, and spatial presentation of information creates a discourse that makes the presented information more meaningful. In other systems with entertainment as an objective, such as the Virtual Storyteller
[11], the aim is to completely generate an entertaining story rather than represent existing content.

Different methods of narrative generation often fall into two types; grammar narratives, and emergent narratives. Grammar narratives work by modeling the rules of a given genre and using structuralism to create a grammar of narrative elements. A discourse is then generated by fitting prewritten narrative segments together using the rules of the grammar. An example of such a system is Artequakt [2] and, to an extent Card Shark [6]. In contrast emergent narratives generate a story by presenting a simulation of the story setting, often using agents to play the parts of characters within a story that follow the rules of the environment and using a director agent to influence the actor agents into a creative narrative. Examples of emergent narratives are Façade [9] and the Virtual Storyteller [11].

Existing techniques often succeed in generating narratives but they have several drawbacks. Narratives generated from story grammars are heavily bound to the rules of a given genre and become very formulaic, and emergent narratives can seem like a bland account of a set of actions as the generation is based on a simple report of what happened in sequence, and as such lacks emphasis and flavor. Both techniques generate narratives that can tend to lack any authorial voice, leading to narratives without any emphasis, creating stories without an objective that can seem directionless. A human author imbeds meaning, subtle themes, and their own goals into a piece - these are lacking in any computer generated narratives. If direction, emphasis, or the authorial voice could be incorporated into generated narratives then it would lead to less bland or formulaic stories.

\section{THEMATIC SYSTEMS}

\subsection{Thematic Model}

In previous work [8] we proposed a thematic underpinning to narrative generation in the form of a thematic model that described how themes are constructed within a narrative. The thematic model is largely based on Tomashevsky's work on thematics. The foundation of the model(as shown in Figure 2). It describes narratives as being built of natoms (narrative atoms) which contain features that denote motifs which in turn connote themes.

For example, we might view a digital photo as a natom, and the tags on that photo as the features that denote a particular motif. Thus a photo tagged with 'daffodil' could denote the motif of 'flower', which connotes the theme of 'spring'. Themes can themselves build up into new themes, for example the theme of 'christmas' can be used to connote the theme of 'winter'.

\section{THEMATIC BUILDER PROTOTYPE}

In order to evaluate the effectiveness of the model a prototype system was built that utilised an instance of the model. The prototype uses the model to select images from Flickr that have strong relevance to particular themes. The prototype went under the working name of the Thematic Model Builder (TMB).

This instance of the model was built in xml and four themes were modeled and expanded (all sub themes and motifs were modeled as well): winter, spring, celebration, and family. The process of defining an instance of the model for 


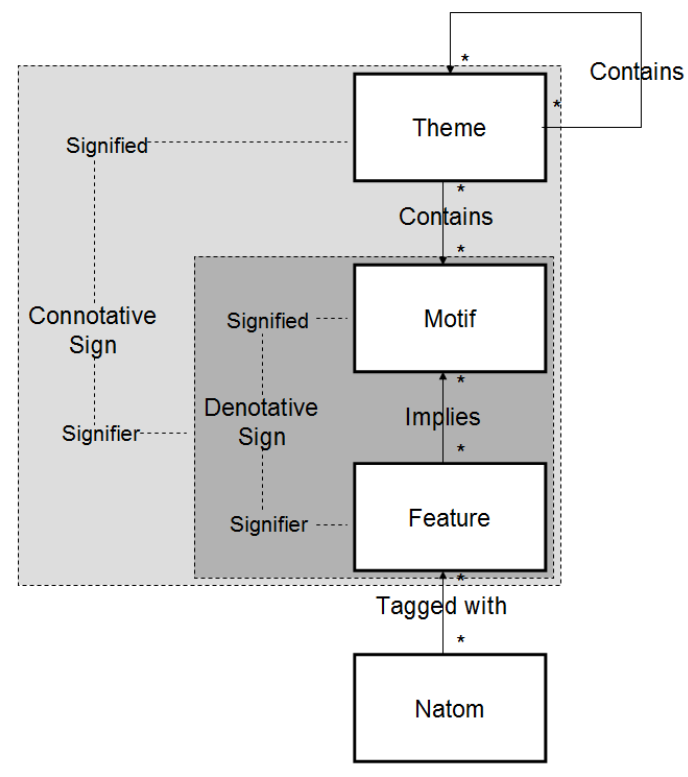

Figure 2: The Thematic Model

particular themes is a complex and subjective one [8]. We explored a systematic method for building themes based on semiotics. Initially we identify what connotes that theme, these connotative signs will make up the themes sub themes and motifs. However, these signs become sub-themes only if all of the aspects of their concept in turn connote the theme being built, otherwise the sign should become a seperate theme in its own right. Thematic objects anchored to a particular device within the narrative become motifs which have their features defined by likely tags that dennote the object.

The prototype itself was written in java with a simple JSP front end. For the purposes of this prototype and evaluating the model, Flickr was chosen as a source of natoms. As a folksonomy its items have rich semantic annotations in metadata [1] that make the features in each image apparent and it has a large freely available body of resources. The library of images (the fabula) was generated by making a keyword search of Flickr on the desired subject and storing the top $\mathrm{n}$ images (where $\mathrm{n}$ is the desired size).

The system then followed an algorithm of measuring the thematic quality of each natom in the fabula. It returns the natoms with the highest scores according to two metrics:

- Component coverage: the proportion of high-level subthemes or motifs that a natom has features for - this is useful for measuring how strongly a natom matches the desired theme. (for example, winter expands several high-level sub-theme and motifs including christmas, snow and cold. A natom matching just one of these has less coverage than one that matches many)

- Thematic coverage: the proportion of desired themes that a natom has features for - this is useful for searches with multiple themes

The TMB Prototype allows us to compare the effectiveness of selecting photos according to their theme with the process of selecting photos based directly on their tags.

\section{EVALUATION}

\subsection{Experiment Design}

For the evaluation it was important to measure what advantage there was in using a thematic system for natom selection over a keyword search system, but we also wanted to see whether themes emerged more strongly from groups of images than with individuals.

The evaluation asked participants to rate images individually and in sets according to how they matched a given subject and theme (for example, 'London in Winter'). The images and sets were generated in four different ways:

- TMB: Using the TMB and Flickr API to search by subject and select by component coverage

- Flickr: Using Flickr to search by subject and theme, filtered by relevance

- BaseL(ow): Selecting images from Flickr at random

- BashH(igh): Using Flickr to search tags by subject and filter manually

In this way we hoped to compare the performance of the TMB with keyword search on Flickr, and place both of these methods in context by comparing them to random and handpicked samples. For each test the user would be presented with two titles and under each the images for the test (depending on the test either individually or in groups) and asked to rate them 1-5 on their relevance to the title. To ensure the data was representative we chose titles composed of contrasting themes and fabulas as well as well matched themes and fabulas. We also included titles that included more then one theme in seperate tests.

In order to make the evaluation fair we presented the single image text first (so participants would not already have associated them with a group). The images on the single image test were also randomly shuffled and for the group tests we randomised the order in which sets appeared. We also added a restriction on image groups that no more than one image would be allowed per author - this is because image sets published by an author naturally flow and would artificially seem to be stronger montages. Finally users were only allowed to take the evaluation once, a unique evaluation link for each user was given out per email address.

Each test contained two titles composed of different subjects and themes from the four the TMB prototype was able to use, in each test one title paired the theme with a complementing fabula, the other title paired the theme with a contrasting fabula to observe performance under different conditions. The titles chosen for single themes were London in Winter, Celebration and Earthquake, Spring Picnic, and Family Factory and for multiple themes My Family in New York at Winter, and Celebrating the New House in Spring.

Our pilot study was performed with 22 users. While this is a relatively low number of people it still gave us a large amount of data, as each user was asked to rate 40 images and 4 groups for each of the 4 sources. This resulted in 880 data points for single images and 88 for groups, enough for quantitive significance to emerge (which we measured with a t test). 


\subsection{Evaluation Results}

The data from the pilot evaluation show some significant results. The mean rating of natoms from the TMB is higher then that for a keyword search (Flickr) in both single and group images. Figure 3 and Tables 1 and 2 show the data and t-tests for single images. Figure 4 and Tables 3 and 4 show the data and t-tests for grouped images. The hypothesis that the TMB selects natoms more relevant to the title then a keyword search is true with only a 2.5 percent probability of error for both group and single images.

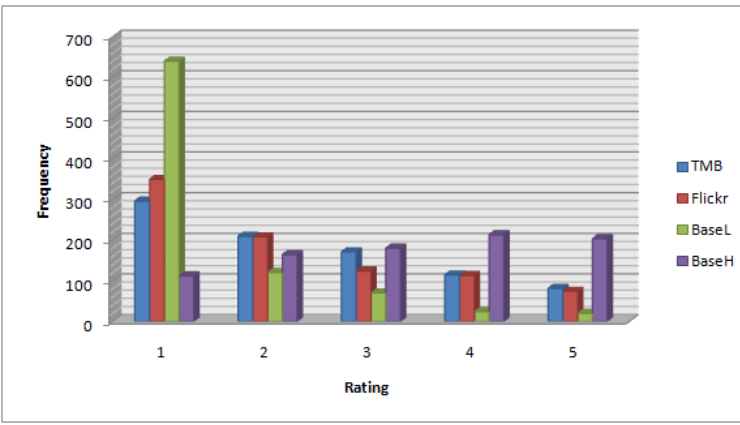

Figure 3: Single Image Rating Frequency

\begin{tabular}{|l|l|l|l|l|l|l|}
\hline Set & 1 & 2 & 3 & 4 & 5 & Total \\
\hline TMB & 296 & 209 & 171 & 114 & 81 & 871 \\
\hline Flickr & 349 & 208 & 124 & 113 & 74 & 868 \\
\hline BaseL & 640 & 120 & 70 & 24 & 20 & 874 \\
\hline BaseH & 112 & 164 & 180 & 213 & 203 & 872 \\
\hline
\end{tabular}

Table 1: Single Images Rating Frequency

\begin{tabular}{|l|l|l|l|}
\hline Set & Mean & SD & Variance \\
\hline TMB & 2.397 & 1.319 & 1.741 \\
\hline Flickr & 2.257 & 1.330 & 1.769 \\
\hline BaseL & 1.471 & 0.926 & 0.850 \\
\hline BaseH & 3.264 & 1.345 & 1.809 \\
\hline \multicolumn{2}{|r|}{$\mathrm{t}=2.207, \mathrm{df}=1737, \mathrm{p}=0.025$}
\end{tabular}

Table 2: Single Images Rating Satistics

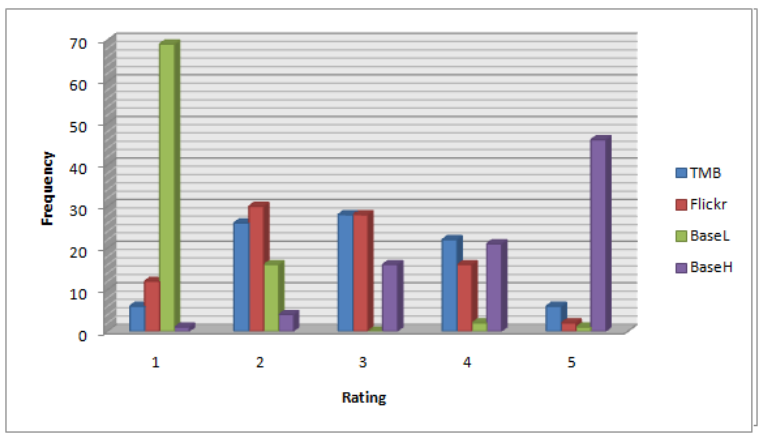

Figure 4: Grouped Image Rating Frequency

At first glance the difference between the TMB and Flickr only appears to be slight however it must be seen in the context of the difference in results between a best case scenario

\begin{tabular}{|l|l|l|l|l|l|l|}
\hline Set & 1 & 2 & 3 & 4 & 5 & Total \\
\hline TMB & 6 & 26 & 28 & 22 & 6 & 88 \\
\hline Flickr & 12 & 30 & 28 & 16 & 2 & 88 \\
\hline BaseL & 69 & 16 & 0 & 2 & 1 & 88 \\
\hline BaseH & 1 & 4 & 16 & 21 & 46 & 88 \\
\hline
\end{tabular}

Table 3: Grouped Images Rating Frequency

\begin{tabular}{|l|l|l|l|}
\hline Set & Mean & SD & Variance \\
\hline TMB & 2.955 & 1.049 & 1.101 \\
\hline Flickr & 2.614 & 1.011 & 1.021 \\
\hline BaseL & 1.295 & 0.697 & 0.486 \\
\hline BaseH & 4.216 & 0.976 & 0.953 \\
\hline \multicolumn{2}{|r|}{$\mathrm{t}=2.182, \mathrm{df}=172, \mathrm{p}=0.025$}
\end{tabular}

Table 4: Grouped Images Rating Satistics

(human selection: BaseH) and a worst case scenario (random selection: BaseL). Figures 5 and 6 show the relevant means and standard deviations in a way that they can be compared. These ranges are rather smaller than we might expect, and in this context the improvement given by TMB is rather more impressive.

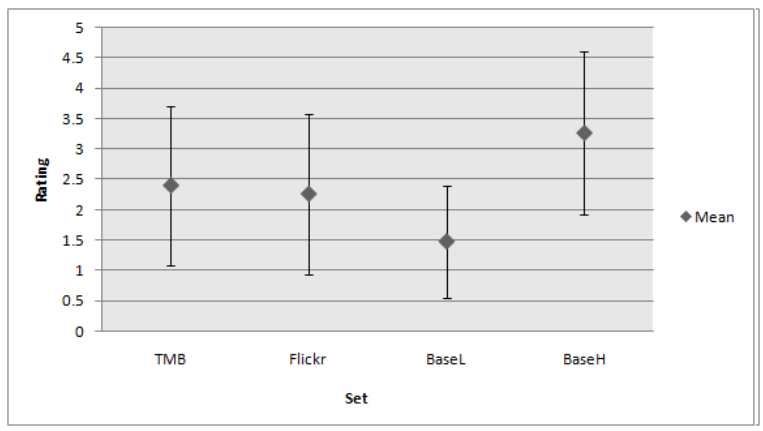

Figure 5: Single Image Mean and Std. Dev.

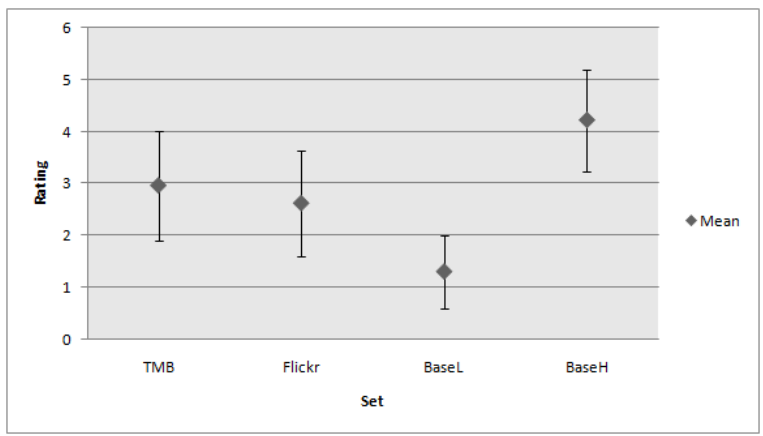

Figure 6: Grouped Image Mean and Std. Dev.

As expected the results also show that the TMB proves significantly better in a montage context where it can build themes over a group of natoms, a t-test shows this hypothesis to be true with only a 0.05 percent probability of error. In addition the data shown in table 5 reveals that while both a keyword search and TMB improved when their natoms were presented as a group the TMBs improvement was much more significant, the hypothesis that the TMBs improvement was 
greater then the improvement of a keyword search in a group context is shown with this data to be true according to a ttest with a 0.5 percent probability of error.

\begin{tabular}{|l|l|l|l|}
\hline Set & Mean & SD & Variance \\
\hline TMB & 0.553 & 1.419 & 2.013 \\
\hline Flickr & 0.351 & 1.436 & 2.060 \\
\hline \multicolumn{4}{|c}{$\mathrm{t}=2.949, \mathrm{df}=1737, \mathrm{p}=0.005$}
\end{tabular}

\section{Table 5: Grouped Images Improvement Statistics}

These results offer encouraging observations towards two of our evaluation objectives. The TMB seems to be performing better then a keyword search with some significance and further more it seems the TMB is very strong within a group context, this could lead us to believe it could perform similarly strongly within a narrative context however a full evaluation would be necessary to confirm these initial quantitative findings as well as answer further evaluation objectives to refine the process of calculating thematic quality.

\section{CONCLUSION AND FUTURE WORK}

In this paper we have described our efforts to build a thematic system that can improve photo montages by giving them a cohesive theme. We have presented an initial evaluation of the prototype that aimed at comparing our thematic approach with simple filtering based on metadata tags.

The analysis of our results shows that the thematic system consistently provides better images than simple keyword filtering, and that this improvement is small but significant for both single images, and images that are evaluated in a collection. Our results also show that images in a collection are more effective at communicating themes, and that the improvement of using a thematic system is more pronounced when using collections. This indicates that a thematic system could have a positive role in narrative generation in terms of enriching the generated narratives, providing that the thematic choice is appropriate for the media available, and that it is aligned with the purpose of the narrative.

It is our intention to take this work forward with a largerscale evaluation, and to use this new data to explore correlations between the thematic properties of chosen images (e.g. do they focus on one or many themes) and their ability to communicate those themes to a viewer. We also intend to investigate how the system behaves with requests that have multiple themes as well as themes that conflict with their desired fabula. We hope to use this information to improve our thematic system, before exploring its impact on more sophisticated narrative structures.

Narrative generation systems offer a potential solution to the problem of information overload, and could help people make more sense of the many information sources around them. We believe that incorporating a thematic system into the narrative generation process could produce narratives that are more purposeful and cohesive, and which seem richer and more engaging as a result.

\section{REFERENCES}

[1] H. Al-Khalifa and H. Davis. Folksonomies versus automatic keyword extraction: An empirical study. IADIS International Journal On Computer Science And Information Systems (IJCSIS), 1:132-143, 2006.

[2] H. Alani, S. Kim, D. Millard, M. Weal, W. Hall, P. Lewis, and N. Shadbolt. Automatic ontology-based knowledge extraction and tailored biography generation from the web. IEEE Intelligent Systems, $18: 14-21,2003$.

[3] M. Alberink, L. Rutledge, and M. Veenstra. Sequence and emphasis in automated domain-independent discourse generation. In Information systems, pages $1-10,2003$.

[4] R. Barthes and L. Duisit. An introduction to the structural analysis of narrative. New Literary History, 6:237-272, 1975

[5] H. Berghel. Cyberspace 2000: dealing with information overload. Commun. ACM, 40(2):19-24, 1997.

[6] M. Bernstein. Card shark and thespis: exotic tools for hypertext narrative. In Proceedings of the twelfth ACM conference on Hypertext and Hypermedia, 2001.

[7] P. DeBra, A. Aerts, B. Berden, B. de Lange,

B. Rousseau, T. Santic, D. Smits, and N. Stash. Aha! the adaptive hypermedia architecture. In Proceedings of the fourteenth ACM conference on Hypertext and hypermedia, pages 81-84, 2003.

[8] C. Hargood, D. Millard, and M. Weal. A thematic approach to emerging narrative structure. In $W e b$ Science at Hypertext08, 2008.

[9] M. Mateas and A. Stern. Façade: An experiment in building a fully-realized interactive drama. In Game Developers Conference, 2003.

[10] M. McQuillan. The Narrative Reader. Routledge, London, 2000.

[11] M. Theune, S. Faas, A. Nijholt, and D. Heylen. The virtual storyteller: Story creation by intelligent agents. In TIDSE 2003: Technologies for Interactive Digital Storytelling and Entertainment, 2003.

[12] B. Tomashevsky. Russian Formalist Criticism: Four Essays, chapter Thematics, pages 66-68. University of Nebraska Press, 1965.

[13] M. Tuffield, S. Harris, D. P. Dupplaw, A. Chakravarthy, C. Brewster, N. Gibbins, K. O'Hara, F. Ciravegna, D. Sleeman, Y. Wilks, and N. R. Shadbolt. Image annotation with photocopain. In First International Workshop on Semantic Web Annotations for Multimedia (SWAMM 2006) at WWW2006, Edinburgh, United Kingdom., 2006. 